\title{
Evaluation of Rate and Time to Arteriovenous Fistula Maturation in Hemodialysis Patients
}

\author{
Syed Zia Ul Haq ${ }^{1,}$, , Musa Bin Bashir ${ }^{2}$, Mirwais Khan ${ }^{3}$, Maivand Khan ${ }^{1}$, Abdullah ${ }^{1}$ \\ ${ }^{1}$ Faculty of Pharmacy and Health Sciences, University of Balochistan, Quetta, Pakistan \\ ${ }^{2}$ Xi' an Jiaotong School of Medicine Xi'an University, Xi'an, China \\ ${ }^{3}$ Balochistan Institute of Nephrology and Urology Quetta, Quetta, Pakistan
}

Email address:

assad.pharmacist@gmail.com (S. Z. Ul Haq)

*Corresponding author

\section{To cite this article:}

Syed Zia Ul Haq, Musa Bin Bashir, Mirwais Khan, Maivand Khan, Abdullah. Evaluation of Rate and Time to Arteriovenous Fistula Maturation in Hemodialysis Patients. American Journal of Nursing Science. Vol. 10, No. 5, 2021, pp. 222-226. doi: $10.11648 /$ j.ajns.20211005.12

Received: August 29, 2021; Accepted: September 11, 2021; Published: September 27, 2021

\begin{abstract}
The acceptance rate of hemodialysis (HD) in end stage renal disease (ESRD) patients has been increasing globally. Pakistan is a CKD high burden country with the increasing acceptance rate of HD. Unfortunately, there is dearth of published information regarding the rate, time to and factors associated with AVF maturation. The current retrospective cohort study was conducted with the aim to evaluate the rate, time to and factors associated with delayed AVF maturation. This study was conducted at the HD unit of Baluchistan Institute of Nephrology-Urology Quetta, Pakistan. The record of 120 HD patients who underwent native arteriovenous fistula (AVF) creation was reviewed focusing on the associated factors and arteriovenous fistula maturity. A standardized data collection form was used to abstract patients' socio-demographic and clinical data. Delayed AVF maturation was defined as AVF which got mature after six weeks of its creation. Statistical Package for Social Sciences (SPSS version 20) was used for data analysis. Categorical data was presented at frequencies and percentages whereas, continuous data was presented as mean \pm standard deviations. Chi-square and Fisher exact tests wherever applicable were conducted to find association between patients' socio-demographic and clinical characteristics and delayed AVF maturation. A p-value $<0.05$ was considered statistically significant. Mean age and body mass index of the study participants were respectively $43.20 \pm 15.184$ years and $20.14 \pm 4.37 \mathrm{~kg} / \mathrm{m}^{2}$. Majority of them were males $(53.3 \%)$, belonged the age group $31-45$ years $(42.5 \%)$, rural residents $(62.5 \%)$ and non-smokers $(65.8 \%)$. A total of $93.3 \%$ patients were suffering from hypertension and $6.7 \%$ suffered from diabetes mellitus. Majority of the study participants had no family history of CKD (81.7\%) and baseline visit of dialysis they had CKD duration of 2-5 years (47.5\%). The mean time to AVF maturation in the current cohort was $41.9 \pm 8.5$ days. A total of $91.7 \%$ patients achieved the early maturation of AVF. No factor had statistical significant association with delayed AVF maturation. The rate of AVF maturation in the current cohort is comparatively high than reported in the published literature. Being a single centre study with retrospective design its results cannot be generalized. In the current study, $91.5 \%$ of the patients achieved early maturation of AVF. The rate of early maturation in the current cohort was comparatively higher than that reported in the previously published literature. In this retrospective study, we didn't find out any factor which had significant association with time to AVF maturation.
\end{abstract}

Keywords: Hemodialysis, Fistula, Maturation, Pakistan

\section{Introduction}

Chronic kidney disease (CKD) is defined as damage of kidney or glomerular filtration rate (GFR) $<60 \mathrm{~mL} / \mathrm{min} / 1.73$ $\mathrm{m}^{2}$ for 3 months or more, irrespective of cause [1] Renal replacement therapy (RRT) replaces the normal blood filtering function of the kidney. When both the kidneys are not working well (also known as renal failure) the RRT is used [2]. Renal replacement therapy includes dialysis (hemodialysis or peritoneal dialysis), hemofiltration, and hemodiafiltration, which are various ways of filtration of 
blood with or without machines. Renal replacement therapy also includes kidney transplantation, in which the old kidney is replaced by a donor kidney [3].

Kidney transplantation is the organ transplant of a kidney into a patient with end-stage renal disease [4]. It is typically classified as deceased-donor (known as cadaveric) or livingdonor transplantation depending on the source of the donor organ [5]. Dialysis continue to be the most frequent type of RRT because of the low rate of renal transplantation, strictly related to insufficient organ donation to meet demand [6]. As a consequence, there is increasing demand of hemodialysis among ESRD patients [7].

Hemodialysis, commonly known as kidney dialysis, is a process of purifying the blood of a person whose kidneys are not in a state of purifying the blood normally [8]. This type of dialysis achieves the extracorporeal removal of waste products such as creatinine urea and free water from the blood when the kidneys are not working or in a state of kidney failure [9]. Due to highly prevalence of CKD in Quetta [10] this research can prove to contribute efficiently to science hence the present study is aimed to Evaluate the rate and time to arteriovenous Fistula maturation in hemodialysis Patients in Quetta.

\section{Methods}

\subsection{Study Design and Study Settings}

Retrospective cohort study design was adopted to conduct this study at the Baluchistan Institute of Nephrology, Urology Quetta (BINUQ) Pakistan. The study site is the only public sector tertiary care hospital for renal diseases and has the wide catchment area of the whole province and nearby Afghanistan.

\subsection{Study Period}

The study was carried out over period of four months. The data was collected from June 2018 to September 2018. The study population involved adult hemodialysis patients were receiving hemodialysis at the study site.

\subsection{Inclusion Criteria}

Adult age between 18 to 70 years;

No previous intervention at the upper limb;

First time AVF creation.

\subsection{Exclusion Criteria}

Central venous stenosis (pre-existing stenosis);

Severe peripheral vascular disease;

Vascular access site deformity (trauma, hematoma etc).

\subsection{Ethical Considerations}

Ethical approval was obtained from Research and Ethics Committee of Faculty of Pharmacy and Health Sciences University of Balochistan Quetta, Pakistan.

\section{Results}

\subsection{Socio-demographic Characteristics of Study Participants}

Socio-demographic characteristics of the total 120 patients included in the current study are given in table 1. Mean age and body mass index of the study participants were respectively $43.20 \pm 15.184$ years and $20.14 \pm 4.37 \mathrm{~kg} / \mathrm{m}^{2}$. Majority of them were males $(53.3 \%)$, belonged to the age group 31-45 years $(42.5 \%)$, rural residents $(62.5 \%)$ and nonsmokers $(65.8 \%)$.

Table 1. Socio-demographic characteristic of the study participants.

\begin{tabular}{lll}
\hline Variables & Mean \pm SD & No. $(\%)$ \\
\hline Gender & & $56(46.7)$ \\
Female Male & $64(53.3)$ \\
Age (years) & \\
$<18$ & $43.20 \pm 15.184$ & $9(7.5)$ \\
$18-30$ & $11(9.2)$ \\
$31-45$ & $51(42.5)$ \\
$46-65$ & $39(32.5)$ \\
$>65$ & $10(8.3)$ \\
\hline
\end{tabular}

Table 1. Continued.

\begin{tabular}{lll}
\hline Variables & Mean \pm SD & No. $(\%)$ \\
\hline Residence & & \\
Rural & & $75(62.5)$ \\
Urban & $45(37.5)$ \\
Body mass index $\left(\mathrm{kg} / \mathrm{m}^{2}\right)$ & $20.14 \pm 4.37$ & \\
Underweight $(<18.5)$ & & $54(45.0)$ \\
Overweight $(>25)$ & $49(40.8)$ \\
Normal $(18.5-25)$ & $17(14.2)$ \\
Smoking status & \\
Active & $8(6.7)$ \\
Ex-smokers & $33(27.5)$ \\
Non-smokers & $79(65.8)$ \\
\hline
\end{tabular}

CKD, chronic kidney disease; SD, standard deviation.

Table 2. Patients clinical characteristics.

\begin{tabular}{lll}
\hline Variables & Mean \pm SD & No. $(\%)$ \\
\hline Comorbidities & & $112(93.3)$ \\
Hypertension Diabetes mellitus & & $8(6.7)$ \\
Family History of CKD & \\
No & & $98(81.7)$ \\
Yes & & $22(18.3)$ \\
CKD disease duration (years) & $3.36 \pm 2.47$ & \\
$<2$ & & $37(30.8)$ \\
$2-5$ & & $57(47.5)$ \\
$>5$ & & $26(21.7)$ \\
\hline
\end{tabular}

$\mathrm{CKD}$, chronic kidney disease; SD standard deviation.

\subsection{Clinical Characteristics of Study Participants}

Table 2 presents the clinical characteristics of study participants. All of the patients were suffering from comorbidities. A total of $93.3 \%$ patients were suffering from hypertension and $6.7 \%$ suffered from diabetes mellitus. Majority of the study participants had no family history of 
CKD (81.7\%) and baseline visit of dialysis they had CKD duration of 2-5 years $(47.5 \%)$.

\subsection{Baseline Laboratory Data of Study Participants}

Patients' laboratory findings are presented in Table 3 . The mean serum creatinine, blood $\mathrm{Hb}$ and blood urea nitrogen levels of the patients were respectively $7.78 \pm 1.97 \mathrm{mg} / \mathrm{dL}$, $8.83 \pm 1.69 \mathrm{~g} / \mathrm{dL}$ and $144.92 \pm 43.01 \mathrm{~g} / \mathrm{dl}$.

Table 3. Patients'laboratory values.

\begin{tabular}{lll}
\hline Variable & Mean \pm SD & No. $(\%)$ \\
\hline Serum creatinine $(\mathrm{mg} / \mathrm{dL})$ & $7.78 \pm 1.97$ & \\
Blood urea nitrogen $(\mathrm{g} / \mathrm{dl})$ & $144.92 \pm 43.01$ & \\
Hemoglobin $(\mathrm{g} / \mathrm{dl})$ & $8.83 \pm 1.69$ & \\
$>12$ & & $4(3.3)$ \\
$10-12$ & & $22(18.3)$ \\
$7-10$ & & $74(61.7)$ \\
$<7$ & & $20(16.7)$ \\
Red blood cells $\left(\times 10^{6}\right.$ cells/dL) & $3.49 \pm 0.77$ & \\
Hematocrit $(\%)$ & $26.16 \pm 6.26$ & \\
\hline
\end{tabular}

Table 3. Continued.

\begin{tabular}{lll}
\hline Variable & Mean \pm SD & No. (\%) \\
\hline Blood calcium & $8.21 \pm 1.03$ & \\
Normal $(8.5-10)$ & & $78(65)$ \\
Hypocalcemia $(<8.5)$ & & $42(35)$ \\
\hline
\end{tabular}

$\mathrm{gm} / \mathrm{dL}$, gram/deciliter; SD, standard deviation.

Table 4. Site of AVF creation.

\begin{tabular}{lll}
\hline Site of AVF & Frequency & Percentage \\
\hline Right & & \\
Radiocephalic & 9 & 7.5 \\
Brachiocephalic & 3 & 2.5 \\
Brachiobasilic & 4 & 3.3 \\
Left & & \\
Radiocephalic & 61 & 50.8 \\
Brachiocephalic & 32 & 26.7 \\
Brachiobasilic & 11 & 9.2 \\
\hline
\end{tabular}

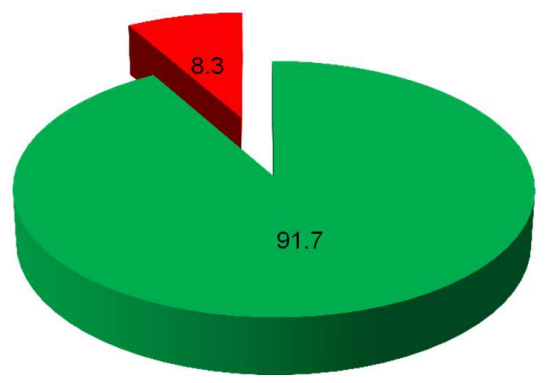

Figure 1. AVF maturation.

\subsection{Time to AVF Maturation}

The mean time to AVF maturation in the current cohort was $41.9 \pm 8.5$ days. A total of $91.7 \%$ patients achieved the early maturation of AVF i.e. the AVF get matured in them within six weeks after creation. While in $8.3 \%$ patients it took longer than six weeks (figure 1).
Patients' socio-demographic characteristics were crosstabulated against time to AVF maturation. The results revealed no statistical significant association between any variable and time to AVF maturation (Table 5).

Table 5. Cross-tabulation between patients' socio-demographic characteristics and AVF maturation.

\begin{tabular}{|c|c|c|c|}
\hline \multirow{2}{*}{ Variables } & \multicolumn{2}{|c|}{ AVF maturation } & \multirow{2}{*}{ p-value } \\
\hline & Early & Delayed & \\
\hline Gender & & & 0.749 \\
\hline Female & $52(92.8)$ & $4(7.2)$ & \\
\hline Male & $58(90.6)$ & $6(9.4)$ & \\
\hline Age (years) & & & 0.299 \\
\hline$<18$ & $11(100)$ & $0(0)$ & \\
\hline $18-30$ & $45(88.2)$ & $6(11.8)$ & \\
\hline $31-45$ & $37(94.4)$ & $2(5.6)$ & \\
\hline $46-65$ & $10(100)$ & $0(0)$ & \\
\hline$>65$ & $7(77.8)$ & $2(22.2)$ & \\
\hline Residence & & & 0.113 \\
\hline Rural & $65(86.7)$ & $10(13.3)$ & \\
\hline Urban & $42(93.3)$ & $3(6.7)$ & \\
\hline Body mass index $\left(\mathrm{kg} / \mathrm{m}^{2}\right)$ & & & 0.138 \\
\hline Underweight $(<18.5)$ & $51(94.4)$ & $3(5.6)$ & \\
\hline Overweight $(>25)$ & $41(87.3)$ & $8(12.7)$ & \\
\hline Normal (18.5-25) & $15(88.2)$ & $2(11.8)$ & \\
\hline Smoking status & & & 0.082 \\
\hline Active & $8(100)$ & 0() & \\
\hline Ex-smokers & $27(81.8)$ & $6(18.2)$ & \\
\hline Non-smokers & 75 (94.9) & $4(5.1)$ & \\
\hline
\end{tabular}

\subsection{Cross-tabulation Between Patients'Socio-demographic Clinical Characteristics and Delayed AVF Maturation}

Patients' clinical characteristics were cross-tabulated against time to AVF maturation. The results revealed no statistical significant association between any variable and time to AVF maturation (Table 6).

Table 6. Cross-tabulation between patients' clinical characteristics and AVF maturation.

\begin{tabular}{|c|c|c|c|}
\hline \multirow{2}{*}{ Variables } & \multicolumn{2}{|c|}{ AVF maturation } & \multirow{2}{*}{ p-value* } \\
\hline & Early & Delayed & \\
\hline Comorbidities & & & 1.000 \\
\hline Hypertension & $102(91.1)$ & $10(8.9)$ & \\
\hline Diabetes mellitus & $8(8.0)$ & $0(0)$ & \\
\hline CKD disease duration (years) & & & 0.116 \\
\hline$<2$ & $33(89.2)$ & $4(10.8)$ & \\
\hline $2-5$ & $55(96.5)$ & $2(3.5)$ & \\
\hline$>5$ & $22(84.6)$ & $4(15.4)$ & \\
\hline Body mass index $\left(\mathrm{kg} / \mathrm{m}^{2}\right)$ & & & 0.138 \\
\hline Underweight $(<18.5)$ & $51(94.4)$ & $3(5.6)$ & \\
\hline Overweight $(>25)$ & $41(87.3)$ & $8(12.7)$ & \\
\hline Normal (18.5-25) & $15(88.2)$ & $2(11.8)$ & \\
\hline Smoking status & & & 0.082 \\
\hline Active & $8(100)$ & 0() & \\
\hline Ex-smokers & $27(81.8)$ & $6(18.2)$ & \\
\hline Non-smokers & $75(94.9)$ & $4(5.1)$ & \\
\hline
\end{tabular}

*Fishser exact test; gm/dL, gram/deciliter; mg/dL, milligram/deciliter; CKD, chronic kidney disease. 
Table 6. Continued.

\begin{tabular}{|c|c|c|c|}
\hline \multirow{2}{*}{ Variables } & \multicolumn{2}{|c|}{ AVF maturation } & \multirow{2}{*}{ p-value* } \\
\hline & Early & Delayed & \\
\hline Hemoglobin (g/dl) & & & 0.570 \\
\hline$>12$ & $4(100)$ & $0(0)$ & \\
\hline $10-12$ & $20(90.9)$ & $2(9.1)$ & \\
\hline $7-10$ & $66(89.2)$ & $8(10.8)$ & \\
\hline$<7$ & $20(100)$ & $0(0)$ & \\
\hline Blood calcium level (mg/dl) & & & 0.730 \\
\hline Normal (8.5-10) & $72(92.3)$ & $6(7.7)$ & \\
\hline Hypocalcemia $(<8.5)$ & $38(90.5)$ & $4(9.5)$ & \\
\hline
\end{tabular}

\section{Discussion}

The current chapter describes the significant findings regarding the time to and factors associated with early maturation of AVF. Autogenous arteriovenous fistula is beneficial in long term haemodialysis access with cost effective and as well as low rate of infection and thrombosis [11]. Previous studies conducted elsewhere have reported a wide range of AVF failure which differs from centre to centre. The failure rate of native AVF has a wide range and differs from centre to centre. In relation to this, numerous studies have been performed in the attempt to correlate the factors responsible for delayed maturation of autogenous AVFs. Pakistan is a high burden CKD country and has a large number of patients on haemodialysis, but there was lack published information regarding time to and factors associated with AVF maturation. To the bets of our knowledge this is the first study which evaluated the time and factors associated with early AVF maturation in haemodialysis patients in Pakistan.

In current study the mean age of the study participant was $43.20 \pm 15.184$ years. The study subjects were comparatively younger than that reported by a study conducted in Malaysia, where the mean age of the study participants was 58.51 years [12]. Progression of CKD can be halted by early diagnosis which can delay the initiation of hemodialysis. The comparatively younger population of the current study advocates the delayed diagnosis of CKD in advanced stage. The delayed diagnosis of CKD and other asymptomatic conditions like hypertension may be partly attributed to the fact that the health care systems in developing countries like Pakistan are directed toward providing symptomatic treatment. It has been reported that an average Pakistani adult visits a primary care physician $4-5$ times each year, but $64 \%$ of adults have never had their blood pressure measured, and $70 \%$ of patients with hypertension and $50 \%$ with DM are unaware of their condition [13]. Majority of current study participants were males, similar high proportion of male patients 56\% have been reported by Malaysia study [14].

In the current retrospective analysis of subjects with native AVF placement, we observed that delayed AVF maturation occurred in only $8.3 \%$ in our center. The prevalence of delayed AVF maturation in this study is much lower than what had been reported by studies conducted elsewhere. In contrast to the lower rate of delayed AVF maturation in the current study
(8.3\%), Wan Najmi et al. from Malaysia reported that 32.3\% of the AVF failed to mature at all [15]. High rates of AVF maturation failure have been reported by other studies too. Miller et al. has reported The prevalence of high rate of delayed maturation in our center, the factors were complex and despite the influence of surgeon experience and adequacy of vessel, there were socio-demographic, comorbidities and biochemical factors influence the fistula maturity. The differences may be due to these following reasons; i) different criteria for determine timing of arteriovenous fistula maturation, ii) low sample size employed by this study ( $\mathrm{n}=$ 100). From what have been observed from our study subjects, we noticed that the timing of referring for AVF creation would rather late with mean serum creatinine 699.82 (403.88) in delayed AVF maturation. This would be the alarming reason prompt to look into in our study subjects. The results of both studies are almost similar to each other. In this study, we didn't find the mean age associated as a significant factor with early arteriovenous fistula (AVF) maturation. In another similar study which also found that the mean age was not associated as a significant factor in the early and delayed arteriovenous fistula maturation [16]. Which reviled that the results in both studies are similar. Same as above the gender is not associated as a significant factor for the early and delayed maturation of arteriovenous fistula (AVF) in this study. In another older study in which the gender is also not found to be a significant factor associated to the early and late maturation of arteriovenous fistula (AVF) [9] which is also similar to our finding as the age.

In this cohort study, we didn't find the body mass index as significant factor associated with the early and late maturation of arteriovenous fistula (AVF) similarly the older study also didn't body mass index as a significant factor associated with early and late maturation of arteriovenous fistula [12]. As a result, both studies show similar results.

In this study of our findings a total of $91.7 \%$ achieved the early maturation of arteriovenous fistula (AVF). According to another similar study conducted in Malaysia the prevalence of early maturation of arteriovenous fistula maturation were $49 \%$ [17] which is much laser than over study. Some of the reasons behind this could be the variation in their design, definitions of the arteriovenous fistula, and some clinical factors are also considered.

In our study, we didn't find any significant factor associated with the early and late maturation of the arteriovenous fistula. This may be due to small sample size and lack of such needed parameters to evaluate these significant factors but some previous studies evaluate such factors that are associated with the early and delayed arteriovenous fistula maturation (AVF) i.e. calcium phosphate, blood urea, dyslipidaemia and diabetes has a central role in the early and delayed maturation of arteriovenous fistula.

In the previous study the calcium phosphate, blood urea and dyslipidaemia are the significant factors that are associated with the delayed arteriovenous fistula maturation.

In recent study, we find that calcium phosphate has a key role in delaying the maturation of arteriovenous fistula. The 
major role of evaluated phosphate in promoting osteogenic and chondrogenic differentiation of vascular smooth muscle contraction (VSMC). Whereas evaluated calcium has a predominant role in promoting the vascular smooth muscle contraction [13].

\section{Conclusions and Recommendations}

To the best of our knowledge this is the first study in Pakistani settings which evaluated the time to and factors associated with AVF maturation in haemodialysis patients. In the current study, $91.5 \%$ of the patients achieved early maturation of AVF. The rate of early maturation in the current cohort was comparatively higher than that reported in the previously published literature. In this retrospective study, we didn't find out any factor which had significant association with time to AVF maturation. The high rate of early AVF maturation and comparatively small number of study subjects in the current study could be the major reason for not finding any significant association.

\section{Limitations}

It was a single centre retrospective study, the sample size of our study was small and also lack of lab parameters. So, its result cannot be generalized. A multi-centre study with a prospective design is recommended to conform the finding of present study.

\section{References}

[1] Eckardt, K.-U., et al., Definition and classification of CKD: the debate should be about patient prognosis - a position statement from KDOQI and KDIGO. American journal of kidney diseases, 2009. 53 (6): p. 915-920.

[2] Fleming, G. M., Renal replacement therapy review: past, present and future. Organogenesis, 2011.7 (1): p. 2-12.

[3] Pannu, N., et al., Renal replacement therapy in patients with acute renal failure: a systematic review. JaMa, 2008. 299 (7): p. $793-805$.

[4] Jorge, A., et al., Kidney Transplantation and Cardiovascular Events Among Patients with End - Stage Renal Disease due to Lupus Nephritis: A Nationwide Cohort Study. Arthritis Care \& Research, 2021.
[5] Knoll, G. A., Kidney transplantation in the older adult. American Journal of Kidney Diseases, 2013. 61 (5): p. 790797.

[6] Téblick, A., et al. "Does Perioperative Patient Perfusion Obviate the Need for Kidney Machine Perfusion?" A Retrospective Analysis of Patients Receiving a Kidney From "Donation After Circulatory Death" Donors. in Transplantation Proceedings. 2020. Elsevier.

[7] Piccoli, G. B., et al., What we do and do not know about women and kidney diseases; questions unanswered and answers unquestioned: reflection on World Kidney Day and International Woman's Day. Physiology international, 2018. 105 (1): p. 1-18.

[8] Thijssen, S., F. Kappel, and P. Kotanko, Absolute blood volume in hemodialysis patients: why is it relevant, and how to measure it. Blood purification, 2013. 35 (1-3): p. 63-71.

[9] PARAB, S. R., HAEMODIALYSIS. 2019.

[10] Rind, H. U. and M. Khan, Etiology and Outcomes of Acute Kidney Injury in Patients Admitted to a Single Tertiary Care Hospital: Balochistan Institute of Nephrology-Urology Quetta. Pakistan Journal of Kidney Diseases, 2020. 4 (4).

[11] Siddiqui, M. A., et al., Predictive parameters of arteriovenous fistula maturation in patients with end-stage renal disease. Kidney research and clinical practice, 2018. 37 (3): p. 277.

[12] Huey Tean, K., Factors associated with delayed arteriovenous fistula maturation in chronic kidney disease patients. 2015, Pusat Pengajian Sains Perubatan, Universiti Sains Malaysia.

[13] Rajput, R., et al., Prevalence, Risk Factors and Management of Anaemia In Non-Dialysis Chronic Kidney Disease Patients: Findings from A Single Centre Study in Pakistan.

[14] Bashar, K., et al., Arteriovenous fistula in dialysis patients: factors implicated in early and late AVF maturation failure. The Surgeon, 2016. 14 (5): p. 294-300.

[15] Siddiqui, M. A., S. Ashraff, and T. Carline, Maturation of arteriovenous fistula: analysis of key factors. Kidney research and clinical practice, 2017. 36 (4): p. 318.

[16] Nguyen, M. C. and K. Heindl-Rusai, Evaluation of HLA typing data and transplant outcome in pediatric renal transplantation.

[17] Lok, C. E., et al., Risk equation determining unsuccessful cannulation events and failure to maturation in arteriovenous fistulas (REDUCE FTM I). Journal of the American Society of Nephrology, 2006. 17 (11): p. 3204-3212. 